\title{
Masking disrupts recovery of location information
}

\author{
D. J. K. MEWHORT, M. FRANK HUNTLEY, and HILARY DUFF-FRASER \\ Queen's University, Kingston, Ontario, Canada
}

\begin{abstract}
In two experiments, we tested subjects' ability to localize a letter in a character string with identification controlled by varying the delay of a mask, dimming the display, or deleting some of the dots used to define the letters on the cathode-ray screen. The first experiment involved two tasks. In the first task, subjects indicated whether or not a letter named verbally had been present in an eight-letter target string. In the second task, they localized a letter named verbally in the target string; the target string was presented by using display parameters shown in the first task to hold character identification between $70 \%$ and $75 \%$ correct. In the second experiment, we tallied errors in a partial-report bar-probe study after equating performance across the manipulations of display quality. Masking disrupted subjects' ability to recover location information more than either a manipulation of stimulus luminance or a manipulation of its visual form.
\end{abstract}

Both anatomical and neuropsychological evidence suggest that location and identity information are processed separately (Gross \& Mishkin, 1977; Schneider, 1969; Trevarthen, 1968; Ungerleider \& Mishkin, 1982; for a recent review see Goodale \& Milner, 1992). The identitylocation distinction is acknowledged in current models of visual processing at either the feature or the object level. Treisman and Paterson (1984), for example, describe perceptual illusions that reflect position errors at the feature level (see also Butler, Mewhort, \& Browse, 1991). Other models consider position errors at the object level. For example, Mewhort, A. J. Campbell, Marchetti, and J. I. D. Campbell (1981) have shown that the pattern of errors in the partial-report bar-probe task reflects loss of information about the position of the letters.

In backward masking, perception of a target is impaired by presentation of a subsequent stimulus positioned to overprint the first. Two kinds of mechanism have been proposed to explain the impairment (Scheerer, 1973). Integration mechanisms explain masking in terms of a loss of data reflecting a mask-induced change in the signalto-noise ratio. Interruption mechanisms, by contrast, explain masking in terms of a disruption to the processes that use the data. In both cases, the idea is that a mask affects a subject's ability to develop identity information, either by diluting the information on which identification is based or by stopping the identification process.

It is clear that masking affects item identification, but the effect of masking on position information is less clear. One reason is that position errors at the feature level are

This research was supported by a grant from the Natural Science and Engineering Research Council of Canada to the first author. Address correspondence to D. J. K. Mewhort, Department of Psychology, Queen's University, Kingston, Ontario, Canada K7L 3N6 (e-mail: doug@vip. psych.queensu.ca). easy to confuse with errors produced by loss of features; both yield errors of identification.

The present experiments were designed to study the possibility that masking disrupts spatial and identity information separately at the object level. In the first experiment, we compared three ways of degrading a visual display of characters. In each trial, subjects were shown an eightletter array tachistoscopically. The array was followed by an auditory probe consisting of the name of a letter. The display quality was degraded by reducing the brightness of the display, by omitting some of the dots used to define the characters, or by using a pattern mask.

With each degrading technique, the subjects performed two tasks. In the first, they were asked to decide whether or not the letter named by the auditory probe was in the array. The display quality was adjusted so that accuracy was held between $70 \%$ and $75 \%$ correct. Once the quality parameters had been set, the task was changed; the subjects were asked to name the probed letter's location. The idea was to reveal potential differences among the three techniques for degrading a display by examining localization under conditions that equated identification.

\section{EXPERIMENT 1}

\section{Method}

Subjects. Thirty volunteers from Queen's University participated in the study. All the subjects had normal or corrected-to-normal vision.

Apparatus and Materials. A PDP/11-23 computer controlled the experiment. The target stimuli were 256 eight-letter consonant strings presented on a Tektronix display monitor (Model 604) equipped with P4 phosphor. The verbal probes were presented over a speaker situated in front of the subject just below the subject's line of vision. The probes were created by a National Semiconductor speech system (the Digitalker, Model DT1053).

Each target string was created by sampling 19 uppercase consonants randomly without replacement. The letters " $J$ " and " $K$ " were not used because pilot subjects had trouble discriminating them 
when presented by the speech system. The mask was composed of a string of eight ampersands aligned to cover corresponding letter positions in the target strings. A fixation stimulus was constructed by displaying a small, dim dot.

The visual characters were constructed by brightening dots within a $7 \times 5$ matrix. The matrix subtended a visual angle of approximately $0.26^{\circ}$ (horizontally) $\times 0.46^{\circ}$ (vertically), and, at normal intensity, the letters appeared blue-white (about $4.4 \mathrm{~cd} / \mathrm{m}^{2}$ ) on a dark screen. The visual angle subtended by the eight-character string was about $2.49^{\circ} \times 0.46^{\circ}$. The monitor was housed in a partially darkened room ( 3.5 lux).

Procedure. The subjects were assigned randomly to three groups of 10 , defined by three display-quality manipulations-masking, dot deletion, and brightness reduction. In all three groups, the subjects performed two tasks. In both tasks, an eight-letter target display was present for $50 \mathrm{msec}$ and was followed immediately by a verbal probe. In the first task, an identification task, the probe letter was present in the visual display on $50 \%$ of the trials, and the subjects had to indicate whether or not the probe had appeared in the display. In the second task, a localization task, the probe letter was always present in the visual display, and the subjects were asked to indicate its position within the stimulus array. The display quality in the localization task was set at a level determined in the identification task to yield approximately $70 \%$ correct.

Before each trial, the subjects were shown a fixation cross displayed at the center of the tachistoscope screen. When ready, they pushed a button to start the trial. After pushing the start button, there was a 10 -msec pause before the target array was shown. The subjects controlled the intertrial interval.

For the subjects in the mask group, the target string was followed, after a variable interval, by a mask composed of eight ampersands displayed for $50 \mathrm{msec}$. The intensity of both the target string and the mask was set at the normal value (about $4.4 \mathrm{~cd} / \mathrm{m}^{2}$ ), and display quality was manipulated by varying the interval between the target and the mask. For those in the luminance group, there was no mask, and display quality was manipulated by altering the intensity of the target display by varying the voltage used to brighten the dots in the target string. In the final group, there was no mask, the intensity was set at normal value (about $4.4 \mathrm{~cd} / \mathrm{m}^{2}$ ), and display quality was manipulated by removing randomly selected dots from the matrix defining each letter.

On each trial of the identification task, the subjects received eight blocks of 16 trials. The probe was present in half of the trials in each block, once in each position in the array. The order of the trials within each block was randomized independently for each subject.

After each block, the experimenter adjusted the display quality to bring performance to between $70 \%$ and $75 \%$ correct. In the mask condition, the experimenter adjusted the interval between the target and the mask; in the dot-deletion condition, he adjusted the percentage of the dots normally used to define the characters. In the luminance condition, he varied the voltage used to brighten the dots defining the stimuli.

On each trial of the location task, the subjects received eight blocks of 16 trials. The probe was present in all trials, and the subjects were required to name the probe's position in the array. The display quality was set at the value determined by the subject's performance in the identification task. The subjects indicated the probe's position by using the digits from 1 to 8 to index positions from left to right.

\section{Results}

Table 1 summarizes identification performance on the first task. The table shows accuracy over the last three blocks of trials (i.e., the last 48 trials) as a function of the display-quality manipulations. Performance across the last three blocks was stable $[F(2,54)<1]$ and equivalent
Table 1

Percentage of Letters (With Standard Deviations) Identified Correctly as a Function of Display-Quality Manipulation and Block

\begin{tabular}{|c|c|c|c|c|c|c|c|}
\hline \multirow[b]{3}{*}{ Block } & \multicolumn{6}{|c|}{ Display-Quality Manipulation } & \multirow[b]{3}{*}{$M$} \\
\hline & \multicolumn{2}{|c|}{ Mask } & \multicolumn{2}{|c|}{ Luminance } & \multicolumn{2}{|c|}{ Dot-Degraded } & \\
\hline & $\%$ & $S D$ & $\%$ & $S D$ & $\%$ & $S D$ & \\
\hline 6 & 71.9 & 9.0 & 73.1 & 17.2 & 73.1 & 17.2 & 72.7 \\
\hline 7 & 73.8 & 15.0 & 72.5 & 11.5 & 78.1 & 7.9 & 74.8 \\
\hline 8 & 68.1 & 11.9 & 75.0 & 13.5 & 74.4 & 13.0 & 72.5 \\
\hline$M$ & 71.2 & & 73.5 & & 75.2 & & \\
\hline
\end{tabular}

across the three manipulations of display quality $[F(2,27)$ $<1]$. Orthogonal contrasts showed no difference in identification with masking compared with the average of the other two conditions $[F(1,27)<1]$ or between the latter two conditions $[F(1,27)<1]$. Finally, the interaction between blocks and conditions was not significant $[F(4,54)<1]$.

Performance in the localization task was assessed by using a measure of contingent uncertainty (see Garner, 1962, chap. 3). We used the uncertainty measure because it gives subjects partial credit for near-miss errors (see Garner, 1962, pp. 67-69).

Each subject's responses were cast into a confusion matrix, $R_{s, r}$, where $s$ indexes the position of the letter in the display and $r$ indexes the position indicated by the subject. Contingent uncertainty, $U(s: r)$, for each subject was computed separately for each quality manipulation:

$$
U(s: r)=U(s)+U(r)-U(s, r),
$$

where

$$
\begin{aligned}
& U(s)=\Sigma-p_{s} \log _{2} p_{s}, \\
& U(r)=\Sigma-p_{r} \log _{2} p_{r},
\end{aligned}
$$

and

$$
U(s, r)=\Sigma \Sigma-p_{s, r} \log _{2} p_{s, r} .
$$

Table 2 summarizes the scores by condition. The maximum score is 3.0 bits, based on eight equally likely positions (i.e., $\log _{2} 8=3$ ). As is shown in the table, accuracy of localization with a mask was lower than with the luminance and dot-deletion techniques $[F(1,27)=5.6534$, $p<.01]$. Accuracy of localization did not differ in the

Table 2

Contingent Uncertainty as a Function of Display-Quality Manipulation and Subject

\begin{tabular}{cccc} 
& \multicolumn{3}{c}{ Display-Quality Manipulation } \\
\cline { 2 - 4 } Subject & Mask & Luminance & Dot-Degraded \\
\hline 1 & 0.995 & 1.052 & 1.448 \\
2 & 1.251 & 1.169 & 1.731 \\
3 & 1.226 & 0.824 & 1.315 \\
4 & 1.218 & 1.435 & 0.912 \\
5 & 1.117 & 1.075 & 1.422 \\
6 & 1.148 & 1.075 & 1.510 \\
7 & 1.431 & 1.589 & 1.389 \\
8 & 0.794 & 1.176 & 1.356 \\
9 & 0.885 & 1.630 & 1.272 \\
10 & 1.038 & 1.693 & 1.289 \\
$M$ & 1.110 & 1.283 & 1.364 \\
\hline
\end{tabular}


luminance and dot-deletion conditions $[F(1,27)<1]$. Thus, even under conditions that equate identification performance, subjects' ability to localize a letter presented in a degraded display depends on how the display has been degraded: Masking the display disrupts the recovery of location information more than either brightness reduction or direct mutilation of the visual image.

\section{EXPERIMENT 2}

In Experiment 1, we examined the effects of three degrading techniques on localization after setting the display conditions to equate accuracy of identification. The results showed that masking caused more spatial disruption than both the luminance and dot-deletion techniques. It appears, therefore, that masking disrupts subjects' ability to recover position information more than the other techniques for degrading the display.

The second experiment was designed to provide converging evidence on the main conclusion. Subjects were shown a row of letters followed by a bar indicating one letter for report (i.e., the same task used by Averbach \& Coriell, 1961). Accuracy in the task was equated by varying the quality of the target display. We used both masking and dot deletion to vary display quality; we used the subjects' errors to measure the kind of difficulty introduced by the two manipulations of quality.

\section{Method}

Subjects. The subjects were 32 undergraduates at Queen's University. All reported normal or corrected-to-normal vision, and all were volunteers.

Apparatus and Materials. The stimuli were eight-letter consonant strings selected at random (without replacement within an eight-letter string).

The letters were displayed using the same font, visual details, and apparatus as in Experiment 1. The probe was an arrow created by attaching two three-dot fins to the bottom of a vertical line. As in Experiment 1, the mask was a string of eight ampersands.

Procedure. The subjects were randomly assigned to two groups of 16 , defined by the display-quality manipulations of dot deletion and masking.

As in the first experiment, a fixation dot appeared at the start of each trial. When ready, the subject pressed a button to continue the trial. After a $10-\mathrm{msec}$ delay, an eight-letter target display was presented for $30 \mathrm{msec}$. Immediately after the target display, a probe indicating one letter for report was presented for $30 \mathrm{msec}$. The probe appeared about $0.23^{\circ}$ above the position of the corresponding letter.

In the masking condition, a mask was presented at a variable interval following the probe. The interval was manipulated by the experimenter to vary display quality. In the dot-deletion condition, the quality of the target display was varied by manipulating the percentage of the normal number of dots used to define the characters. In both cases, display quality was manipulated after each block of 24 trials to bring accuracy to between $55 \%$ and $65 \%$ correct and as close to $60 \%$ as possible.

Each subject received 192 trials, that is, 24 replicates at each letter position. The trials were arranged in eight blocks of 24 trials, and within each block all eight positions were tested three times. A response was required on each trial, and the subjects were warned not to respond with a letter from the wrong part of the display if they were uncertain about the identity of the letter probed. Instead, they were encouraged to respond with a letter that looked like the
Table 3

Percentage of Correct Responses as a Function of Trials and Display Condition

Block of 24 Trials

\begin{tabular}{lcccccccc} 
Condition & 1 & 2 & 3 & 4 & 5 & 6 & 7 & 8 \\
Dot deletion & 60.4 & 60.9 & 62.0 & 60.4 & 61.2 & 62.5 & 58.1 & 62.2 \\
Masking & 59.6 & 60.2 & 62.0 & 62.2 & 63.0 & 63.5 & 63.3 & 62.8 \\
\hline
\end{tabular}

item probed. The subjects were given 24 practice trials prior to the experiment proper.

\section{Results}

Table 3 shows the percentage of letters reported correctly as a function of display-quality manipulation and block of trials. As is clear in the table, the experimenter was able to hold accuracy at about $60 \%$ across trials in both the mask and dot-deletion conditions [for both, $F(7,15)<1.0$ ], and accuracy of report in the two groups was equivalent $[F(1,30)<1.0]$.

The total number of errors was roughly equivalent in the two display-quality conditions. The kind of error in the two cells differed dramatically. In the masking cell, $71.8 \%$ of the errors were intraarray errors; in the dotdeletion cell, by contrast, only $54.6 \%$ of the errors were intraarray errors $[F(1,30)=15.61, p<.001]$. Intraarray errors primarily reflect difficulty in localization; the subjects reported a letter from the wrong position (see Mewhort, Butler, Feldman-Stewart, \& Tramer, 1988; Mewhort et al., 1981). Hence, we take the difference in the kind of error as evidence that masking produces difficulty in localization over and above its role in reducing identification.

\section{DISCUSSION}

In the first experiment, we equated subjects' ability to identify letters across three methods for degrading stimuli (masking, changing the luminance, and changing the visual form) and then tested their ability to localize a designated letter. Masking yielded more localization errors than the other two methods. In the second experiment, we equated performance in a standard bar-probe task under two of the methods for degrading the display (dot deletion and masking) and then examined the nature of the subjects' errors. Even though the total number of errors was constant, masking yielded a greater percentage of intraarray errors (i.e., location errors) than did dot deletion. Both experiments converge on the same conclusion: Masking disrupts recovery of location information at the object level.

Given that masking disrupts location information at the object level, a detailed explanation of how the mask works remains elusive. The problem is that we have no consensus on how the position of an object is represented. Van der Heijden (1992) links an object's position to the position of its constituent features. On that view, an object's position is calculated by reference back to the position of 
its constituent features. If so, a mask could disrupt information about an object's position by interfering with its constituent features or by breaking the backward links. Rueckl, Cave, and Kosslyn (1989) argue that the position of an object is calculated from the position of its features, but, once the calculation has been made, the position of an object is represented independently of its features. A similar idea has been advanced by Mewhort, Marchetti, Gurnsey, and A. J. Campbell (1984). Given an independent representation, a mask could disrupt either the feature data on which the calculation is based or the spatial representation at the object level. Finally, Mozer's (1991) BLIRNET model uses a six-layer detector network; the size of each detector's receptive field increases from the bottom to the top of the hierarchy so that position information is lost as information ascends the hierarchy. Because a mask replaces the features of the display, it impairs the system's ability to recover position information. All three models provide plausible possibilities, but a clear consensus about the architecture of the system is needed before it is possible to come to a decision about how masking disrupts spatial information at the object level.

\section{REFERENCES}

Averbach, E., \& Coriell, A. S. (1961). Short-term memory in vision. Bell System Technical Journal, 40, 309-328.

Butler, B. E., Mewhort, D. J. K., \& Browse, R. A. (1991). When do letter features migrate? A boundary condition for feature-integration theory. Perception \& Psychophysics, 49, 91-99.

GARNER, W. R. (1962). Uncertainty and structure as psychological concepts. New York: Wiley.

Goodale, M. A., \& MilNer, A. D. (1992). Separate visual pathways for perception and action. Trends in Neuroscience, 15, 20-25.

Gross, C. G., \& Mishkin, M. (1977). The neural basis of stimulus equivalence across retinal translation. In S. Harnad (Ed.), Lateralization in the nervous system (pp. 109-122). New York: Academic Press.

Mewhort, D. J. K., Butler, B. E., Feldman-Stewart, D., \& Tramer, S. (1988). "Iconic memory," location information, and the bar-probe task: A reply to Chow. Journal of Experimental Psychology: Human Perception \& Performance, 14, 729-737.

Mewhort, D. J. K., Campbell, A. J., Marchetti, F. M., \& CampBELL, J. I. D. (1981). Identification, localization, and "iconic memory": An evaluation of the bar-probe task. Memory \& Cognition, 9, 50-67.

Mewhort, D. J. K., Marchetti, F. M., Gurnsey, R., \& Campbell, A. J. (1984). Information persistence: A dual-buffer model for initial visual processing. In H. Bouma \& D. G. Bouwhuis (Eds.), Attention and performance X: Control of language processes (pp. 287-298). Hillsdale, NJ: Erlbaum.

Mozer, M. C. (1991). The perception of multiple objects: A connectionist approach. Cambridge, MA: MIT Press.

Rueckl, J. G., Cave, K. R., \& Kosslyn, S. M. (1989). Why are "what" and "where" processed by separate cortical visual systems? A computational investigation. Journal of Cognitive Neuroscience, 1, 171-186.

SCHEERER, E. (1973). Integration, interruption, and processing rate in visual backward masking. Psychologische Forschung, 36, 71-93.

SCHNEIDER, G. E. (1969). Two visual systems. Science, 163, 895-902.

Treisman, A. M., \& Paterson, R. (1984). Emergent features, attention, and object perception. Journal of Experimental Psychology: Human Perception \& Performance, 10, 12-31.

Trevarthen, C. (1968). Two mechanisms of vision in primates. Psychologische Forschung, 31, 299-337.

UNGERLEIDER, L. G., \&ISHKIN, M. (1982). Two cortical visual systems. In D. J. Ingle, M. A. Goodale, \& R. J. W. Mansfield (Eds.), Analysis of visual behavior (pp. 549-586). Cambridge, MA: MIT Press.

VAN DER Heideen, A. H. C. (1992). Selective attention in vision. London: Routledge.

(Manuscript received February 27, 1992; revision accepted for publication June 7, 1993.) 